\title{
Teaching Usability Principles with Patterns and Guidelines
}

\author{
Kostas Koukouletsos, Babak Khazaei, Andy Dearden, Mehmet Ozcan \\ Communications and Computing Research Centre \\ Sheffield Hallam University, \\ Sheffield, UK \\ +441142254951 \\ ccostas@teipir.gr \\ \{B.Khazaei, A.M.Dearden, M.B.Ozcan\}@shu.ac.uk
}

\begin{abstract}
We present a comparative study of the effectiveness of patterns and guidelines as aids to teaching web interaction design. We recruited two groups of novice designers and taught them web design from scratch using a popular authoring tool. We used two balanced sets of guidelines and patterns that in essence present the same advice in two different formats. After the initial training, subjects learned about usability and usability principles with the help of one of these sets. The groups then engaged in two common activities that professional designers must perform, that is designing and evaluating web sites. The final design artefact that was delivered in all tests was a working web site. Here we present the quantitative results for the design phase of this experiment. Evaluation of the designs was conducted by three independent evaluators, using defined metrics. We conclude that both patterns and guidelines help with the design of usable sites, however the advice presented using our patterns format had a greater impact on the novice designers' performance than the same advice in our guidelines format.
\end{abstract}

\section{Introduction}

Guidelines are a traditional format for capturing knowledge and experience and presenting advice about interaction design. Recently researchers in Human Computer Interaction (HCI) have started comparing guidelines with patterns, another emerging tool for capturing knowledge and experience. For example, van Welie et al. [1] argue that the main problem of guidelines is that they do not state the context in which they are applied. Mahemoff \& Johnston [2] suggest that guidelines can be conflicting,

Please use the following format when citing this chapter:

Koukouletsos, K., Khazaei, B., Dearden, A. and Ozcan, M., 2009, in IFIP International Federation for Information Processing, Volume 289; Creativity and HCI: From Experience to Design in Education; Paula Kotzé, William Wong, Joaquim Jorge, Alan Dix, Paula Alexandra Silva; (Boston: Springer), pp. 159-174. 
whereas a pattern presents a solution by demonstrating how conflicting forces in a design can be resolved. Pemberton and Griffiths [3] also argue for advantages of design patterns in comparison to guidelines, suggesting that design patterns are presented in a canonical form and provide much richer information than guidelines.

Despite their claimed shortcomings, guidelines remain the most widely accepted form of presenting experience and knowledge. Their durability and endurance are remarkable. Nielsen [4] reports that general guidelines built 20 years ago are still up to $70 \%$ valid and applicable, while $78 \%$ of guidelines from the early days of the web continue to be valid and relevant.

It may be argued that patterns and guidelines are simply two different formats serving as practical tools to present guidance to designers and practitioners about how to build better artefacts [5]. Borchers [6] states that '.. patterns are, above all, a didactic medium for human readers'. This work attempts to compare patterns and guidelines as two alternative formats of providing knowledge and experience to students acting as novice designers.

Previous empirical work on patterns explores the use of patterns in assisting designers to create applications in new domains and how patterns can benefit the design process by generating and communicating ideas between the members of a design team $[5,7]$. Other research compares patterns and guidelines based on the perceived usefulness of the designers that used them $[8,9]$.

Our approach is to develop a balanced set of patterns and guidelines; experiment with them; and finally test their effectiveness by evaluating and rating the design artefacts produced. This approach gives us a better assessment of the strengths and weaknesses of these competing formats, and their value in HCI education.

Section 2 reviews other empirical work on the evaluation of patterns and guidelines for HCI and their use in teaching usability principles, while Section 3 presents the main hypothesis. Section 4 describes the steps taken in defining and formulating a balanced set of patterns and guidelines for our experimental purposes. Section 5 describes the experimental procedure and the teaching approach. Section 6 discusses the results of the experiment and the greater impact of patterns on our subject group, and finally Section 7 gives our concluding comments.

\section{Empirical Work on Patterns and Guidelines in HCI}

Several researchers used and propose patterns as an aid to teaching HCI principles and design concepts. Borchers [10], having used patterns to teach two HCI design courses, suggests that patterns can lead to above average retention of design values, and concludes that patterns have great potential since there are not just interesting as a topic, but also useful as a tool and format to teach basic design principles. Seffah [11] considers patterns useful not only as a design tool, but also as a learning resource part of an educational framework for effective training and improving developers' skills in Human Centred Design. In particular, user interface design patterns are considered useful for teaching students to master visual information design, including screen layout and interaction design. Griffiths and Pemberton [12] used patterns as a means to teach the interaction design of software systems. Three 
different approaches were used: teaching about patterns, teaching through patterns and discovering patterns. Although students had difficulties to discover and suggest new patterns, the authors report that existing patterns improved the level of discussion within the design teams and that patterns helped students to produce an improved final artefact; however they also express the need to verify this impression more formally. Laakso et al. [13] used their teaching experience of interface design courses to develop a collection of 25 user interface patterns and pattern candidates. The authors report that initial designs have consistently improved and in some cases the students were producing high quality interface designs in very short time. Dearden, Finlay \& colleagues, investigated the use of a pattern language as a tool to support participatory design of web-based systems [14-16], demonstrating that a pattern language can benefit a participatory design approach and can help users generate artefacts in a prototype form.

Although quantitative empirical evidence about the usefulness of patterns in the domain of HCI is not extensive [17], recently there have been a few studies examining the use of patterns in real design activities and investigating the problems of applying patterns in practical settings.

Chung et al. [5] evaluated the effectiveness of using patterns with designers in developing applications in the emerging field of ubiquitous computing. The designers were asked to design a location-enhanced application. One group had access to a set of patterns while the other did not. They concluded that experienced designers without patterns performed better than novice designers with patterns, i.e. patterns can not substitute experience, but that experienced designers (although not familiar with this particular domain) performed better when they had patterns available. Saponas et al. [7] investigated the use of patterns in early design activities with designers building a digital home application. The participants were split randomly into two groups and were asked to perform the same design task. Only one group was given access to the set of patterns with the help of a tool within a web browser. The study suggests that team members were able to use the patterns productively to generate new ideas and to exchange design ideas with each other. Furthermore, the designs of the patterns group had a lower mean number of heuristic violations than the control group, implying that the use of patterns promoted higher quality design products. Cowley and Wesson [9] compared patterns and guidelines based on the opinions of two groups of students, one using guidelines and the other patterns, to investigate the usefulness of patterns as a design aid. Based on initial analysis of the students' ratings of their opinion about patterns and guidelines, the authors concluded that designers consider patterns useful for design and an effective and efficient design aid. However, both patterns and guidelines groups felt equally positive in their intention for using them in future projects. Kotze et al. [18] examined the use of patterns and anti-patterns to teach HCI principles to two groups of students. The authors provide statistical results, from assessing the students, indicating that anti-patterns can be counter productive.

Our work can be regarded as a complementary approach to the ones described above. There are however, noticeable differences:

1. We are assessing completed web pages and sites instead of a prototyping task, working with a widely accepted web design tool. 
2. We use a balanced set of patterns and guidelines providing a balanced body of knowledge to the two groups, thus allowing us to compare the effectiveness of the different formats.

3. We engage the subjects into a realistic course spread over a full semester of teaching.

\section{Hypothesis}

The main purpose of our study is to assess the effectiveness of patterns and guidelines on the design habits of novice designers. We follow a matched subjects design, studying web usability principles by means of either a set of patterns or an equivalent set of guidelines. The dependent variable is the scores of the students in a practical design test. Thus our hypothesis is:

H1: There is a difference between the performances of students taught usability principles using patterns, and students taught using guidelines.

The null hypothesis is then that:

H0: There is no significant difference between the performance of the students in the patterns and the guidelines groups.

\section{Balancing Patterns and Guidelines for the Experiment}

\subsection{Patterns and Guidelines for the Study}

Identifying a set of patterns and guidelines that could be implemented as an instructional tool for teaching design principles was critical to the success of our experiment. The following criteria were used for the selection:

1. Patterns and guidelines should be meaningful for the students, close to their technical level and experience, and appropriate to their particular level of knowledge;

2. Patterns and guidelines should be easy to apply in a web site design using existing basic technical web design skills.

Our patterns and guidelines are primarily derived from existing sources: Koyani et al.'s collection [19] for guidelines (hereafter Koyani's guidelines) and two widely known pattern languages $[20,21]$. A quick review of the patterns that we considered for our experiment revealed that many of the patterns in these sources did not meet our criteria. Current patterns and pattern languages [20-22] contain many patterns that are complicated and address complex and high level design issues (e.g. ecommerce, site genres, trust and credibility, etc.). Novice designers do not possess the necessary technical knowledge and experience to apply these patterns in their design work, and such patterns deal with advanced issues that may only be clearly explored in large scale design tasks. Similar observations can be made about the available guidelines: although their complexity is somewhat lower than patterns, many are not suitable for a time limited experiment designed for a novice designer. 
Another problem with existing sets of patterns and guidelines is the lack of consistency between them. This can be attributed to an existing gap between patterns and guidelines in their approach to design: patterns are used mostly as components to build web sites while guidelines as small pieces of prescriptive advice. Comparing, for example, the Koyani guidelines [19] with the currently developed pattern languages [20-22], we observed that only a small number of guidelines could be directly mapped to the existing patterns. A pattern sometimes contains advice that is spread across several guidelines.

Because of these difficulties, and in order to avoid complicated procedures of transforming patterns into guidelines or developing new patterns, with all the drawbacks that such a procedure would imply, we decided to form a body of usable guidelines complying with our criteria and then transform them into patterns, since it was easier to find guidelines that were addressing low level problems.

An additional complication in this experiment was the fact that the work was conducted in a Greek University, hence, since few HCI patterns are available in Greek, patterns and guidelines written in English required translation into Greek to make them accessible for the students.

\subsection{Forming the Sets}

We followed a stepwise approach of defining the set of patterns and guidelines for our experiment.

- Step 1: We initially collected all the patterns from the two pattern languages that met our criteria. There were not many. However we made sure that every pattern that could be used was included in the set. This set included only, what we name as, 'simple patterns' that could be directly mapped to a single guideline each. These patterns were then translated into Greek.

- Step 2: We selected a set of guidelines from the Koyani collection that was appropriate for our study. These were translated into Greek. The chosen set of Koyani's guidelines was transformed into a set of patterns using the format that we employed for this study. Some of the guidelines, relevant to a common design issue, were consolidated and formed a single pattern. We name patterns that correspond to more than one guideline as 'compound patterns'.

- Step 3: Once the set of patterns was finalised, patterns were transformed into guidelines. The patterns that were collected in step 1 were transformed into guidelines. For the rest of the patterns, since there were originated from guidelines the work involved for the transformation was minimal.

We examined all the information and advice included in each pattern and the corresponding guideline (or guidelines) to verify that equivalent information was being given.

\subsection{Pattern Format}

We chose to present our patterns in a format that would make balancing guidelines and patterns easy since we transformed guidelines to patterns. Also, the pattern 
format should be simple for the students to familiarize themselves with the pattern terminology, being introduced for the first time to the idea of patterns. The format is consistent with published formats for web design. We used mainly the formats from van Welie [21] and van Duyne et al. [20] pattern languages to structure our patterns. Patterns were presented in the following format: the name of the pattern; a picture as an example of its application; a section describing when the pattern is used; a short problem statement followed by a detailed description of the problem and the solution to the problem and the forces involved; the solution for the problem in a condensed statement; other patterns that may be taken into account; and finally more examples of the pattern.

Each pattern was formed by taking, to a great extent, the advice and examples given in each guideline and presented them in a pattern format. The advantage of this approach, in connection with the format adopted for patterns and guidelines, is that the basic content, narrative and examples, of the patterns and guidelines are equivalent. The only exception to this rule is the examples section where, in some cases in the 'more examples' section, more examples were included. This is a very distinctive feature of the pattern format and we followed this tradition of presenting patterns. Also, since patterns suggest the use of a pattern language, in each pattern, wherever applicable, in the relative section, links to other related patterns were given.

The patterns that were used for the study were simple. However, developing and implementing simple patterns, draw a parallel with the use of simple programming patterns, inspired by the success of Object Oriented Design (OOD) patterns [23]. OOD patterns focus on advanced software design problems with a target audience mostly designers and are used only in advanced design courses. More recently, related research [24-26] has been carried out into simple programming patterns that are being developed with intention to teach simple programming principles to computer science students. In a similar way, we introduce simple design patterns to teach usability principles and web design issues to novice designers.

\subsection{Guideline Format}

The format that guidelines were presented was adapted from Koyani's guideline format [19]. Essentially, all the information presented in the Koyani guidelines was included. The format consists of a heading-title for the guideline, the guideline itself followed by comments explaining why the guideline should be used and in some cases how it should be applied, and finally examples of the guidelines. The illustrations in the original guideline depicting bad or good examples of web designs were used. However, these examples were placed after the full set of guidelines, at the end of the booklet, as a set of examples about good design, each with the guideline title that the example refers to.

\subsection{Refining the Sets}

The patterns and guidelines evolved through two pilot studies [27-29] and several reviews with evaluators and experienced designers. 
Some of the examples initially used for teaching were not successful in that they did not communicate the indented message to the students. After an extensive search through the Internet, focusing mainly on Greek sites, we were able to find successful and illustrative examples. Subsequently, we either included them in our collection or replaced the inappropriate ones.

Some of the patterns names and guidelines titles were changed to improve the students' understanding. We also added some patterns/guidelines, and modified the focus of others in ways that we believed would help students improve their design habits. These patterns/guidelines dealt with some of the most common mistakes that students made for example with text alignment, fonts and font sizes, links and underlined text.

\subsection{The Final Set of Patterns and Guidelines for the Study}

The final set formulated for the experiment was 35 guidelines and 25 patterns. Some patterns/guidelines that could not be directly implemented in the experimental design tests were included in the sets since they were considered essential for the overall training of student subjects as novice designers.

Table 1 depicts only the guidelines and the corresponding patterns that could be directly implemented and used by the students in the final design tasks (19 patterns and 29 guidelines). Column one identifies 7 broad categories of advice, these being: Home page; Page layout and Design; Navigation; Headings, Titles and Labels; Links; Text and Typography; and Lists. The second column gives the title for each pattern, and column three gives the title to the corresponding guideline or guidelines.

There were 15 'simple' patterns that could be mapped onto their equivalent 15 guidelines, 1 'compound' pattern that corresponded to 2 guidelines and finally 3 'compound' patterns that corresponded to sets of 4 guidelines each.

\section{The Experimental Study}

\subsection{Overview of the Experiment}

We recruited a group of students and taught them web design from scratch using a widely used authoring tool. Once the tutorials about web design finished, a pretreatment assignment was used to split the subjects into two balanced groups. Each group learned about usability and usability principles using either the set of patterns or the set of guidelines.

After this teaching, subjects engaged in two common activities professional designers are involved, that is designing and evaluating tasks. More qualitative data was also collected through interviews after completion of the task. This data will be reported elsewhere. 
Table 1. Categories, patterns and corresponding guidelines

\begin{tabular}{|c|c|c|}
\hline Category & Pattern Name & Guideline Title \\
\hline \multirow{2}{*}{ Home Page } & 1. Home Page Link & 1. Enable Access to the Homepage \\
\hline & 2. Home Page Length & 2. Limit homepage length \\
\hline \multirow{3}{*}{$\begin{array}{l}\text { Page Layout } \\
\text { \& Design }\end{array}$} & $\begin{array}{l}\text { 3. Consistent } \\
\text { Important Items }\end{array}$ & 3. Place important items consistently \\
\hline & 4. White Space & 4. Use moderate white space \\
\hline & 5. Item Alignment & 5. Align Items on a Page \\
\hline \multirow{3}{*}{ Navigation } & 6. Users' Location & 6. Provide Feedback on Users' Location \\
\hline & $\begin{array}{l}\text { 7. Descriptive Tab } \\
\text { Labels }\end{array}$ & 7. Use descriptive tab labels \\
\hline & 8. Repeated Menu & 8. Repeat Navigation at the Bottom of the Page \\
\hline \multirow{2}{*}{$\begin{array}{l}\text { Headings, } \\
\text { Titles \& } \\
\text { Labels }\end{array}$} & 9. Nice Headings & $\begin{array}{l}\text { 9. Use Unique, Descriptive and Emphasized } \\
\text { Headings }\end{array}$ \\
\hline & 10. Page Titles & 10. Provide descriptive page titles \\
\hline \multirow{4}{*}{ Links } & $\begin{array}{l}\text { 11. Consistent and } \\
\text { Descriptive Links }\end{array}$ & $\begin{array}{l}\text { 11. Provide links where users may need them } \\
\text { 12. Match link names with destination pages } \\
\text { 13. Ensure embedded links are descriptive } \\
\text { 14. Designate used links }\end{array}$ \\
\hline & $\begin{array}{l}\text { 12. Obvious Links - } \\
\text { Consistent Links }\end{array}$ & $\begin{array}{l}\text { 15. Provide consistent clickability cues } \\
\text { 16. Avoid misleading cues to click }\end{array}$ \\
\hline & 13. Clickable Images & 17. Use Text for Links and Clickable Images \\
\hline & 14. To-the-Top Link & $\begin{array}{l}\text { 18. Allow Users to Go Back to the Top of the } \\
\text { Page }\end{array}$ \\
\hline \multirow{4}{*}{$\begin{array}{l}\text { Text \& } \\
\text { Typography }\end{array}$} & $\begin{array}{l}\text { 15. Consistency in } \\
\text { Text }\end{array}$ & $\begin{array}{l}\text { 19. Ensure visual consistency } \\
\text { 20. Format common items consistently } \\
\text { 21. Use at least } 12 \text {-point font } \\
\text { 22. Use familiar fonts }\end{array}$ \\
\hline & 16. Visible Text & $\begin{array}{l}\text { 23. Use Black Text on Plain, High-Contrast } \\
\text { Backgrounds }\end{array}$ \\
\hline & 17. Emphasized Text & 24. Emphasize Importance in Text \\
\hline & 18. Aligned Text & 25. Use Proper Alignment for Text \\
\hline Lists & 19. Lists & $\begin{array}{l}\text { 26. Display Related Items in Lists } \\
\text { 27. Introduce Each List } \\
\text { 28. Format Lists to Ease Scanning } \\
\text { 29. Capitalize First Letter of First Word in Lists }\end{array}$ \\
\hline
\end{tabular}

\subsection{Subject Groups}

The participants for the study were students from the final year of Automation Department, TEI Piraeus, Greece. All of them had already completed three years of study, and they were quite skilful with the use of computers and familiar with internet related activities, but had not previously studied web design.

For the purposes of the experiment, a special course was scheduled for the first half of the Spring Semester 2006. The course included twenty-five plus hours of 
lectures and seminars about Web Design including the use of FrontPage, usability design principles and evaluation techniques. The course was set apart from any regular formal academic courses that the students were following, and had no affect on their marks.

Forty five volunteer students enrolled for the course. Three groups, with fifteen students in each group, were formed and tutorial sessions about web design techniques of two hours duration twice in a week were conducted. Throughout the course, several small assignments enabled the students to practice the techniques presented by the lessons taught. Every student was assigned to one computer, working on his/her own. We ensured that all students received sufficient training through practice by completing, to a great extent, all assignments during the tutorials.

\subsection{Teaching Approach}

Tutorials were conducted by the first author, while a technical assistant was present at all times to help with technical problems and the use of equipment (network, projector screen, etc.).

The tutorials introduced the use of tools and technical issues about web design. One of the tutorials was about general usability principles and evaluation of web sites. This tutorial was delivered before the pre-treatment assignment.

During the tutorials, we intentionally presented and practiced a certain number of design principles and design issues, using traditional teaching techniques without employing patterns or guidelines. That was deemed to be appropriate because we wanted students to advance with web design and to avoid making elementary usability mistakes. We made sure that students had time to practice and adopt these basic design practices found on all contemporary sites.

Apart from these design principles that were delivered to all students, the tutor would not directly suggest any quality or usability issues or how to make improvements to the appearance of a web page since that would directly interfere with quality and usability issues of web design with the individual student. Avoiding presenting advice and good practices about web design was a rule that was strictly followed during the tutorials, since advice and good practices were supposed to be delivered only during the patterns/guidelines lectures.

\subsection{Pre - Treatment Assignment}

Once the initial tutorials finished, a pre-treatment assignment was given to the students that required the design of a small but complete site. For simplicity, and in order to allow students to focus on major design issues rather than complex navigational schemes, the site was one level deep requiring only one main navigation menu. Each page of the web site was based on a fixed width template. This assignment was used as a criterion to split the students into two balanced groups with regards to their design ability and skills.

Based on their performance of the pre-treatment assignment, we categorized the students into 4 groups: adequate, good, very good and excellent. After that, using a 
stratified sampling, we divided the students into 2 balanced groups. In this way, one group was equivalent to the other with regards to their ability and knowledge about web design. For the final experiment one group would be exposed to usability issues using patterns and the other one using guidelines.

\subsection{Teaching Patterns and Guidelines}

The set of 35 guidelines and the equivalent set of 25 patterns, as discussed in Section 4, were presented to the two groups. Patterns were presented to one group and guidelines to the other. The students were given a booklet of 33 pages of either patterns or guidelines to use during the lecture and to study afterwards. The time needed to cover the set of guidelines and the equivalent set of patterns was 3 hours. The guidelines/patterns tutorial was given by an independent lecturer while the first author of this paper was acting as an observer. The duties of the observer were to make sure the lecturer did not favour patterns or guidelines and to make sure that every pre-planned aspect of the teaching process was covered.

Finally, a revision test was given to the students, in order to elicit at least a minimum degree of study and comprehension from the students. A two-page form, with a full list of the pattern names or guidelines titles that were presented in the class, was given to each student at the end of the tutorials. Students were asked to study patterns/guidelines and then describe, in their own words, the most essential and critical idea that was incorporated within each pattern/guideline. The forms were delivered, duly completed, either electronically or by hand before the beginning of the design tests.

\subsection{Details of the Design Test}

Out of the initial 45 students taking part in the experiment 39 participated in the design test: 19 in the patterns group and 20 in the guidelines group. There were three design test sessions, lasting 2 hours each. During the sessions, one more lecturer was present to assist with questions and problems that students faced during the task, taking notes while observing students and helping with the smooth process of the experiment.

To limit the possibilities of one student affecting or influencing another, computers and positions of the students were pre-arranged so that a student could only view his own screen. In that way, students were designing using their own ideas and inspiration.

The test was asking students to design a small one level deep web site about a small company hosting and creating web sites for its customers. The site contained five pages and students were required to work and design only the three pages of the site; the rest of the two pages were included in order to make the site conceptually complete and to oblige students to provide a more realistic navigation within the site. Each page of the site was based on a three-column fixed width page layout.

Furthermore in order to compare similar sites, and to avoid evaluating pages with different text and graphics resulting in dissimilar design approaches and formatting, the content, text, and photos/images were provided for each page. The use of a 
template and the partial model, although restricting the creativity of students, supported more consistent evaluations based on defined metrics. The metrics focussed on usability issues that patterns and guidelines dealt with.

A separate instructions sheet was given to each student. The inclusion of specific content together with the instructions given to the students invoked the use of patterns/guidelines presented during the tutorials, and allowed us to measure the impact patterns/guidelines had on their design products.

Students had to format text, pictures, specify the layout, identify and indicate links, provide a navigation scheme using interactive buttons, and in general design according to the instructions and the requirements given. In brief, students were expected to design as best as they could, in order to have the best possible presentation of the company on the Internet, taking into account the purpose and the type of the company, using the usability principles they have learned during the tutorials.

Students were advised not to use any other material apart from that provided already in the site, unless they felt compelled to. Each student had access to a small directory containing certain small images, arrows, bullets small gif files, etc.

Students were free to use their booklet of patterns and guidelines during the tasks. Also, they were allowed to ask any question about the use of tools or how to do certain things in case they had difficulties or they couldn't remember the right procedure. We wanted students to design what they envisioned and imagined and not to be affected by lack technical knowledge.

\subsection{Scoring of the Students}

The evaluation of the subjects' designs was conducted by independent evaluators, using a predetermined set of metrics. Each metric corresponds to one of the patterns (and the matched guideline or guidelines) that were presented to the students. This set of metrics measures the degree to which they followed design principles and advice given by the patterns/guidelines used in the tutorial. Some of our metrics are broken down into sub-components, or sub-metrics, in order to make the task of scoring easier for the evaluators.

Table 2. Example of a metric

\begin{tabular}{|l|l|}
\hline $\begin{array}{l}\text { Metric Name: } \\
\text { N5_HoPaLink }\end{array}$ & $\begin{array}{l}\text { Is there a home link on every page? Do they use the logo as a link to } \\
\text { home page? Or probably any appropriate labels (e.g. 'Home') }\end{array}$ \\
\hline Protocol & $\begin{array}{l}\text { Review all pages and check if there is a home link on every page. } \\
\text { The Home Page itself should count as one of the pages. }\end{array}$ \\
\hline Scores & $\begin{array}{l}\text { If a link to the Home page exists in every page score }=4 \text {; in } 2 \text { pages } \\
\text { score }=2 ; \text { in any } 1 \text { page score=1; Otherwise score=0. Max Score=4. }\end{array}$ \\
\hline
\end{tabular}

Each metric was accompanied by a small explanation giving details regarding scoring and how the evaluator should proceed using the metric. Table 2 depicts one metric with the specific instructions for pattern 'Home Page Link' and the matched guideline 'Enable Access to the Homepage'. 
Each metric score contributes equally to the score of the student. A score from 0 to 9 shows the conformity and application of the patterns/guidelines to the site. For some metrics, a different scale was used in order to facilitate the work of the evaluators. However, at the end all scores were converted to scores in 0-9 scale.

Three independent evaluators (academics with web design experience) were used to view and assess the students' sites according to the predefined set of metrics. Each evaluator spent on average 55 minutes for each site to make an evaluation based on the set of metrics; however the actual time to administer the whole evaluation process was much longer. Most of the times the evaluator worked better if he judged all the sites together working just on one only metric and comparing each page with another one.

The default web page browser on the student's PC was MS Internet Explorer v.6.0.2. An Internet Explorer engine based type browser (Slim Browser V4.06) with multi tab functionality was used by the evaluators. Tabs allow the user to view multiple web pages in the same window and to switch between the web pages with ease and comfort without the need to open a new browser window. The evaluators could afford to open all the pages of the site at the same time or to load a particular page from all students on the same unique browser window. In that way previewing, checking, and comparing web sites and pages was straight forward and evaluation was taking less time.

After the collection of the scores there was a review of the scores with all the members of the team and some errors that were found on the scores were corrected.

\section{Results of the Design Test}

\subsection{Analysis of the Evaluation Scores}

Using the quantitative measures of the three evaluators, we statistically analyzed the performance of the two groups. Students were assessed using the metrics in 7 different dimensions-components of good design practice: Home page; Page layout and Design; Navigation; Headings, Titles and Labels; Links; Text and Typography; Lists.

Table 3. Average percentage scores of students

\begin{tabular}{|c|c|c|c|c|c|c|c|}
\hline & 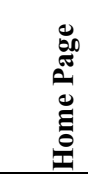 & 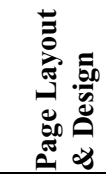 & 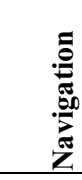 & 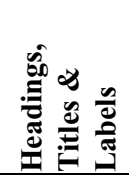 & $\frac{y}{3}$ & 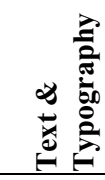 & $\frac{n}{2}$ \\
\hline Guidelines & 59.8 & 81.2 & 67.9 & 65.1 & 50.8 & 85.5 & 49.4 \\
\hline Patterns & 66.4 & 85.0 & 81.5 & 79.1 & 64.3 & 86.4 & 39.8 \\
\hline Difference & 6.6 & 3.8 & 13.6 & 14.0 & 13.5 & 0.9 & -9.7 \\
\hline
\end{tabular}

Table 3 shows the differences between the calculated average scores of the students, in the two groups, for each of the seven measured categories. The average 
scores are given as an average percentage score, where 100 denotes the maximum that could be achieved for the category. Evidently students in the patterns groups performed better overall. Their scores were higher in six out of the seven categories; in the Links category the guidelines group scored better.

The main purpose of the experiment was to assess the effectiveness of patterns and guidelines on the design habits of novice designers. To address this question we compare scores from the 2 groups reflecting the application of usability principles that were conveyed using the two formats (treatments). The dependent variable is the scores of the students. The score for each participant was obtained by adding the points for each metric. Each metric contributed equally to the score. The null hypothesis, as described in Section 3, is that there is no difference in the performance of the groups.

An independent samples t-test (2-tailed) was conducted to test the hypothesis. The patterns group $(\mathrm{M}=128.97, \mathrm{SD}=20.16)$ performed better than the guidelines group $(\mathrm{M}=116.25, \mathrm{SD}=13.66), \mathrm{T}(37)=2.317, \mathrm{P}=0.0261$. Since the computed $\mathrm{P}$-value is less than 0.05 , we can reject the null hypothesis in favour of the alternative and accept that there was a statistically significant difference between the two groups in applying the advice, knowledge and experience conveyed by patterns and guidelines.

The combined scores of the three judges (average score for the particular metric), were used for this analysis. Actually, there were few differences in the scores of the evaluators, and a t-test performed for each individual judge produces similar results, i.e. the null hypothesis is rejected.

\subsection{Subjective and Objective Metrics}

Our metrics can be classified as being objective or subjective. Objective metrics, for example, are those measuring the application of guidelines like 'Provide descriptive page titles', 'Allow users to go back to the top of the page', 'Repeat navigation at the bottom of the page', etc. For the objective metrics all evaluators should be able to give the same scores.

Subjective metrics are those that produce a score depending, to a certain extent, on the judgement of each evaluator. Subjective metrics provide a measure of the application for guidelines like 'Use Moderate White Space', 'Provide Feedback on Users' Location', 'Emphasize Importance', etc.

We used the subjective metrics to produce a separate comparison of the performance between the two groups of students.

Employing an independent samples t-test (2-tailed), the patterns group $(\mathrm{M}=56.89, \mathrm{SD}=14.82)$ was better than the guidelines group $(\mathrm{M}=47.61, \mathrm{SD}=9.88)$, $\mathrm{T}(37)=2.313, \mathrm{P}=0.0264$.

The computed P-value is less than 0.05 , indicating that there was a statistically significant difference between the two groups in favour of the patterns group. 


\section{Summary and Further Work}

We conducted an extensive study in teaching web design with the help of patterns and guidelines. We developed two equivalent sets of web patterns and web guidelines for novice users. We also developed suitable teaching material based on the sets and used them in two pilot studies and one main experimental study. The teaching material and the set of patterns and guidelines were adapted from existing patterns and guidelines. The teaching material can be used to form the basis for teaching design principles and usability to novice designers.

Both sets were simple enough for the students to understand and, what was more important the students had the means and the technical knowledge to fully employ them in order to design a real web site in electronic form using FrontPage. The performance of the students in the patterns group was better than that in the guidelines group, in all but one category, and the overall scores of the students in the patterns group was better that the students in the guidelines group. The difference in the overall scores was statistically significant.

The comparison was based on the ratings of three independent evaluators who used the predefined set of metrics to compare the designs. What is more important is that a comparison made using only the subjective metrics shows that the patterns group performed also significantly better.

All the statistical analysis of the quantitative data that was performed through $\mathrm{t}$ tests and all results indicate that the use of patterns can lead to better performance for novice designers than the use of guidelines and that patterns can have a stronger impact, provided they address design issues close to the level of experience of the students.

Future analysis of the available data could also take into account the scores of the students in the pre-test experiment, so that a comparison between high performing and low performing students can be made. At the same time, analysis of the qualitative data that we have collected through questionnaires and interviews, during the experiment, will complement our quantitative analysis and will shed more light into the effectiveness of patterns and guidelines for novice designers.

We could also look into the effect of compound and simple patterns on the design habits of students. First indication is that the impact of compound patterns is less significant, suggesting that compound patterns are close to the cognitive limitation of novice designers, that is they offer too much knowledge and advice that probably do not create a clear picture of all the usability issues involved.

\section{References}

1. M. van Welie, G. van der Veer, and A. Eliëns, Patterns as Tools for User Interface Design, in: International Workshop on Tools for Working with Guidelines, edited by C. Farenc and J. Vanderdonckt, (Springer-Verlag, London, 2000), pp. 313-324.

2. M. Mahemoff and L.J. Johnston, Usability Pattern Languages: the "Language" Aspect, in: Human-Computer Interaction, INTERACT '01, edited by M. Hirose, (IOS Press, Amsterdam, 2001), pp. 350-358. 
3. L. Pemberton and R.N. Griffiths, Don't Write Guidelines Write Patterns! (cited 2006-1222); http://www.it.bton.ac.uk/staff/lp22/guidelinesdraft.html, (no date).

4. J. Nielsen, Durability of Usability Guidelines, (cited 2006-10-21); http://www.useit.com/ alertbox/20050117.html (2005).

5. E.S. Chung, J.I. Hong, J. Lin, M.K. Prabaker, J.A. Landay, and A.L. Liu, Development and evaluation of emerging design patterns for ubiquitous computing, in: Across the Spectrum: Designing Interactive Systems, DIS2004, (ACM, New York, 2004), pp. 233-242.

6. J.A. Borchers, A Pattern Approach to Interaction Design (John Wiley, Chichester, UK, 2001).

7. T.S. Saponas, M.K. Prabaker, G.D. Abowd, and J.A. Landay, The impact of pre-patterns on the design of digital home applications, in: Designing Interactive Systems, DIS 2006, (ACM, New York, 2006), pp.189-198.

8. J. Wesson and N.L.O. Cowley, Designing with patterns: Possibilities and pitfalls, in: Proceedings of the 2nd Workshop on Software and Usability Cross-Pollination: The Role of Usability Patterns, INTERACT 2003, edited by M. Rauterberg, M. Menozzi and J. Wesson, (IOS Press, 2003).

9. N.L.O. Cowley and J.L. Wesson, An experiment to measure the usefulness of patterns in the interaction design process, in: Lecture Notes in Computer Science 3585 - HumanComputer Interaction - INTERACT 2005, edited by M.F. Costabile and F. Paternó, (Springer, 2005), pp. 1142-1145.

10.J.A. Borchers, Teaching HCI Design Patterns: Experience from Two University Courses, Position paper for Patterns in Practice workshop at CHI 2002, (cited 2007-01-22); http://www.hcipatterns.org/tiki-download_file.php?fileId=19, (2002).

11.A. Seffah, Learning the ropes: human-centered design skills and patterns for software engineers' education, Interactions, 10(5), 36-45 (2003).

12.R.N. Griffiths and L. Pemberton, Teaching Usability Design Through Pattern Language, (cited 2006-10-25); http://www.it.bton.ac.uk/staff/lp22/CHIpaper.html, (no date).

13.K.P. Laakso, A. Saura, and S.A. Laakso, Pattern Languages for Interaction Design, Position paper for CHI 2000 Workshop Pattern Languages for Interaction Design, (cited 2006-11-19); http://www.cs.helsinki.fi/u/salaakso/patterns/, (2000).

14.A. Dearden, J. Finlay, E. Allgar, and B. McManus, Using Pattern Languages in Participatory Design, in: Proceedings of the Participatory Design Conference 2002, edited by T. Binder, J. Gregory, and I. Wagner, (CPSR, Palo Alto, CA, 2002), pp. 104-113.

15.A. Dearden, J. Finlay, L. Allgar, and B. McManus, Evaluating pattern languages in participatory design, in: Adjunct Proceedings of CHI 2002, (ACM Press, New York, USA, 2002), pp. 664-665.

16.J. Finlay, E. Allgar, A. Dearden, and B. McManus, Pattern Languages in Participatory Design, in: People and Computers XVI-Memorable Yet Invisible, Proceedings of HCI2002, edited by X. Faulkner, J. Finlay, and F. Detienne, (Springer-Verlag, London, 2002), pp. $159-174$.

17.A. Dearden and J. Finlay, Patterns Languages in HCI: A Critical Review, HumanComputer Interaction, 21(1), 40-101 (2006).

18.P. Kotzé, K. Renaud, and J. Van Biljon, Don't do this - Pitfalls in using anti-patterns in teaching human-computer interaction principles, Computers \& Education, DOI: http://dx.doi.org/10.1016/j.compedu.2006.10.003, (2006).

19.S.J. Koyani, R.W. Bailey, and J.R. Nall, Research-Based Web Design \& Usability Guidelines (Computer Psychology, USA, 2004).

20.D.K. Van Duyne, J. Landay, and J.I. Hong, The Design of Sites (Addison-Wesley, Boston, MA, 2003).

21.M. Van Welie, Patterns in Interaction Design, (cited 2006 09-14); http://www.welie.com/, (2005). 
22.I. Graham, A Pattern Language for Web Usability (Addison-Wesley, London, 2003).

23.E. Gamma, R. Helm, R. Johnson, and J. Vlissides, Design Patterns: Elements of Reusable Object-Oriented Software (Addison-Wesley, Reading, MA, USA, 1994).

24. J. Bergin, Coding at the Lowest Level - Coding Patterns for Java Beginners, (cited 200607-15); http://pclc.pace.edu/ bergin/patterns/codingpatterns.html, (2006).

25. O. Muller, B. Haberman, and H. Averbuch, (An almost) pedagogical pattern for patternbased problem-solving instruction, in: Proceedings of the 9th Annual SIGCSE Conference on Innovation and Technology in Computer Science Education, (ACM, NY, USA, 2004), pp. 102-106.

26. O. Muller, Pattern oriented instruction and the enhancement of analogical reasoning, in: Proceedings of the 2005 International Workshop on Computing Education Research (ACM, NY, 2005), pp. 57-67.

27.K. Koukouletsos, B. Khazaei, A. Dearden, and D.I. Tseles, Comparing patterns and guidelines in web design, in: Proceedings of the 1st International Scientific Conference eRA2006, (cited 2006-03-25); http://ikaros.teipir.gr/era/ab1.htm, (Tripolis, Greece, 2006).

28.P. Kotzé, K. Renaud, K. Koukouletsos, B. Khazaei, and A. Dearden, Patterns, AntiPatterns and Guidelines-Effective Aids to Teaching HCI Principles?, in: Inventivity: Teaching theory, design and innovation in HCI, Proceedings of HCIEd2006, edited by E.T. Hvannberg, J.C. Read, L. Bannon, P. Kotzé, and W. Wong, Limerick, Ireland, (2006), pp. $115-120$.

29.K. Koukouletsos, Evaluating the effectiveness of guidelines and patterns for web design, in: Proceedings of HCI2005: The Bigger Picture, Volume 2, edited by L. MacKinnon, O.W. Bertelsen, and N. Bryan-Kinns, (BCS, 2005), pp. 209-211. 